\title{
Nivel de concordancia entre personal entrenado y no entrenado en triaje y un sistema informático automatizado
}

\author{
Gabriela Lattuada*, Ricardo Robaina ${ }^{\dagger}$, Julio $\mathrm{Carrau}^{\ddagger}$, Norberto Liñares§, \\ José Gorrasis, Fernando Machado"
}

\section{Resumen}

El triaje -proceso de clasificación de pacientes según prioridades asistenciales- es una herramienta reconocida para la gestión asistencial y administrativa de los departamentos de emergencia(1). En el Hospital de Clínicas, luego de ocho años de funcionamiento, se cuenta con un proceso de triaje automatizado y normalizado que se transformó en la base organizativa para el abordaje calificado de las consultas.

El objetivo del trabajo fue comparar la concordancia del triaje efectuado por personal de salud entrenado y no entrenado previamente sin apoyo informático versus el sistema informatizado, comparándolo con los resultados del mismo en tiempo real. Se observó que existe un mayor nivel de concordancia del personal entrenado con los resultados del sistema informatizado si lo comparamos con el personal no entrenado.

El observador capacitado con más resultados concordantes obtuvo 55,9\% de acuerdos con el sistema informatizado de triaje (19 concordantes de 34), y el que obtuvo menos resultados concordantes tuvo $32,4 \%$ de similitud (11 concordantes de 34). En el grupo de no expertos el promedio global de concordancia fue de $41,5 \%$. El observador experto tuvo $79,4 \%$ (27/34) de resultados iguales y un índice Kappa respecto al sistema informatizado de triaje. El observador experto tuvo un índice Kappa de 0,695, mientras que los observadores capacitados tuvieron un índice Kappa de 0,19 y 0,23 cuando se compararon con el sistema informático y el observador experimentado, respectivamente. Se concluye que un período breve de entrenamiento en triaje no aumenta la concordancia cuando se comparan con los resultados del triaje usando un sistema informático y con el triaje realizado por un observador experimentado. Estos resultados deberían ser validados en series mayores de pacientes.

Palabras clave: Triaje

Estudio comparativo

Procesamiento automatizado de datos
Key words: $\quad$ Triage

Comparative study

Automatic data processing

\footnotetext{
* Asistente del Departamento de Emergencia del Hospital de Clínicas.

† Asistente del Departamento de Estadística del Hospital de Clínicas.

‡ Director de Tecnologías de la Información del Hospital de Clínicas.

$\S$ Prof. Agregado del Departamento de Emergencia del Hospital de Clínicas.

II Prof. Titular del Departamento de Emergencia del Hospital de Clínicas.

Los autores declaran no presentar conflictos de intereses personales ni financieros con la institución ni con la Revista Médica del Uruguay.

Correspondencia: Gabriela Lattuada. Carlos Crocker 2781 bis, Montevideo, Uruguay. Correo electrónico: gabrielalattuada@gmail.com

Recibido: 8/11/17

Aprobado: $7 / 5 / 18$
} 


\section{Introducción}

La atención inicial en el servicio de urgencia hospitalario (SUH) y la percepción del paciente en su acceso mismo dependen en gran parte de la valoración efectuada en el triaje, que no solo contempla la clasificación, sino que incluye otros aspectos como la información y ubicación en el servicio ${ }^{(2)}$. Esta función puede ser realizada por diferentes recursos humanos: médicos, enfermeros y estudiantes de Medicina. Contar con un sistema de triaje es considerado un indicador de calidad de riesgo-eficiencia para los servicios de urgencias hospitalarios y un instrumento para elaborar casuística ${ }^{(3,4)}$.

La Organización Panamericana de la Salud (OPS) recomendó la utilización de un sistema de triaje en todos los centros que tienen un servicio de urgencia para primer, segundo y tercer nivel de atención. Recomienda, además, la utilización de un sistema estandarizado compuesto de cinco niveles de prioridad en la atención, que funcione las 24 horas del día, que tenga un lenguaje común, que sea sistematizado e informatizado y que se adapte a la realidad de cada centro asistencial, teniendo en cuenta los tipos de consultas y la disponibilidad de re$\operatorname{cursos}^{(2,5,6)}$.

Ante las importantes ventajas que aportaría un programa de ayuda a la decisión en el triaje, basado en una escala de triaje moderna y validada, desarrollamos nuestro programa informático de ayuda al triaje basado en el sistema de triaje Manchester ${ }^{(5)}$. Para despejar posibles dudas sobre la conformidad del programa y con la finalidad mejorar su versión, realizamos un estudio piloto para evaluar el grado de concordancia interobservador del programa respecto al triaje realizado por profesionales expertos y no expertos.

Se plantearon como objetivos demostrar la clasificación efectuada por personal entrenado previamente (experto) y no experto, y su concordancia con el sistema informatizado.

\section{Material y método}

En primera instancia se realizó un curso de inducción de clasificación por prioridades asistenciales (triaje) desarrollado por la cátedra del Departamento de Emergencia del Hospital de Clínicas. El mismo tuvo como personal objetivo 17 personas del área de la salud (tres médicos, dos practicantes internos de medicina, ocho auxiliares de enfermería y cuatro licenciados en enfermería) seleccionadas por la autoridad local del hospital solicitante del curso, y que pertenecían a diferentes salas de urgencia y emergencia de centros de asistenciales de primer, segundo y tercer nivel de atención de los departamentos de Paysandú y Artigas, y a quienes consideramos como no expertos por no trabajar previamente con un sistema de triaje normatizado y estructurado. Las personas elegidas participaron activamente en la clasificación de los pacientes que consultan en las puertas de emergencia de primer, segundo y tercer nivel de centros de Paysandú y Artigas, aún sin tener acceso a un sistema informatizado y automatizado como apoyo, ni una capacitación mayor en el tema, sabiendo que en el Hospital de Paysandú se reciben un promedio de 100 consultas diarias, y algunas menos en el Hospital de Artigas. El fundamento de elegir un grupo de no expertos se basa, además, en la posibilidad de extender el sistema a operadores no expertos. La elección de la muestra incluye a los participantes de la jornada de triaje en Paysandú, se trata entonces de una muestra de conveniencia. Al mismo tiempo, acordamos que por lo acotado de la muestra las conclusiones tendrán limitación en cuanto a los resultados. De todas formas este estudio piloto tiene el objetivo de analizar la concordancia del método y plantear sugerencias para un estudio de mayor tamaño y diseñado a tales efectos. En este estudio piloto definimos no experto al individuo que no conoce el funcionamiento del sistema de triaje y que recibe un entrenamiento muy breve antes de realizar el triaje de pacientes que se presentó en este trabajo.

\begin{tabular}{|c|c|c|c|}
\hline & COLOR & Tiempo de respuesta & Área de atención \\
\hline Gravísimo & & 0 minuto & Reanimación \\
\hline Muy grave & & 5 minutos & \\
\hline Observación & & 30 minutos & Consultorios de atención \\
\hline No grave & & 120 minutos & Policlínica de atención de urgencia \\
\hline Diferible & & Más de 120 minutos & En policlínicas fuera del servicio de puerta \\
\hline
\end{tabular}

Triaje en el Hospital de Clínicas. 
Tabla 1. Total de acuerdos de los 17 observadores capacitados versus el sistema informático.

\begin{tabular}{|c|c|c|c|c|c|c|c|}
\hline & \multirow[b]{2}{*}{ Rango de triaje } & \multicolumn{6}{|c|}{ Observadores capacitados } \\
\hline & & 1 & 2 & 3 & 4 & 5 & 0 \\
\hline \multirow{9}{*}{$\begin{array}{l}\text { Sistema } \\
\text { informático }\end{array}$} & 1 & 16 & 8 & 7 & 2 & 0 & 1 \\
\hline & 2 & 17 & 75 & 58 & 16 & 0 & 4 \\
\hline & 3 & 8 & 86 & 96 & 72 & 5 & 5 \\
\hline & 4 & 0 & 3 & 23 & 39 & 3 & 0 \\
\hline & 5 & 0 & 1 & 6 & 13 & 14 & 0 \\
\hline & \multirow[t]{2}{*}{0} & 0 & 0 & 0 & 0 & 0 & 0 \\
\hline & & 41 & 173 & 190 & 142 & 22 & 0 \\
\hline & $\begin{array}{l}\text { Concordancias } \\
\text { esperables por azar }\end{array}$ & 2,42 & 50,89 & 89,41 & 16,71 & 1,29 & 160,71 \\
\hline & $\begin{array}{l}\text { Concordancias } \\
\text { observadas }\end{array}$ & 16 & 75 & 96 & 39 & 14 & 240 \\
\hline \multicolumn{8}{|c|}{$\begin{array}{l}34 \text { casos por } 17 \text { evaluadores es igual a } 578 \text {. } \\
\text { Cada casillero es la suma de concordancias para cada rango evaluado; por ejemplo, en el rango } 1 \text { son iguales a } 16 \text {, en el rango } 2 \text { son iguales } \\
\text { a } 75 \text {, en el rango } 3 \text { son iguales a } 96 \text {, en el rango } 4 \text { son iguales a } 39 \text {, en el rango } 5 \text { son iguales a } 14 \text {. Las concordancias esperables por azar se } \\
\text { calcularon de la siguiente manera (multiplicando el total de cada fila y cada columna correspondiente a cada rango evaluado); concordan- } \\
\text { cias esperables por azar: } 41 \times 34 / 578=2,42 ; 173 \times 170 / 578=50,89 ; 190 \times 272 / 578=89,41 ; 142 \times 68 / 578=16,71 ; 22 \times 34 / 578=1,29 \text {. } \\
\text { Sumatoria de concordancias por azar: } 160,71 . \\
\text { Resultado Kappa }=0,19 \text {. }\end{array}$} \\
\hline
\end{tabular}

Las actividades fueron realizadas los días 27 y 28 de abril de 2017. El curso de inducción consistió en un curso teórico del tema (cuatro horas de duración), para posteriormente invitar a cada uno de los participantes a realizar un ejercicio de clasificación de pacientes en forma de taller, sin soporte informático. El curso teórico abarcó nociones de gestión asistencial de las áreas de emergencia, así como la inducción a la utilización del sistema informatizado. Se presentaron 34 casos iguales a cada uno de los talleristas y se les solicitó que otorgaran un color a cada uno de los casos. Los casos clínicos fueron reales, los pacientes habían consultado en la emergencia del Hospital de Clínicas, pero seleccionados dentro de un conjunto amplio, lo cual determinó efectivamente una selección por conveniencia. No se brindaron datos filiatorios que identificaran a los pacientes. Sí se brindaron los datos habituales utilizados para realizar la clasificación, el síntoma guía (motivo de consulta) y las constantes fisiológicas (factores discriminantes) para cada caso y se solicitó que asignaran un nivel de prioridad a cada uno, según lo aprendido en la exposición teórica asignándole el color correspondiente a la prioridad. Paralelamente, se le solicitó a una persona experta en triaje del Hospital de Clínicas (quien lleva tres años de experiencia, denominada observador 1) que efectuara la clasificación de los 34 casos y sin apoyo informático como el resto del grupo. Finalmente, todos los casos fueron clasificados con el sistema informático automatizado del Hospital de Clínicas.

Los resultados fueron llevados a formato de planilla electrónica tipo Excel, para su análisis ulterior. A partir de los datos generados se realizó el cálculo de concordancia a través del coeficiente de concordancia Kappa entre los no expertos, el experto y el sistema informatizado. Se calculó el coeficiente Kappa de la siguiente manera $=(\Sigma$ concordancias observadas $-\Sigma$ concordancias por azar) / (número de observaciones $-\Sigma$ concordancias por azar).

En cuanto a la elección de un valor de corte Kappa, de acuerdo a la literatura previa se menciona que los Kappa son más altos cuando los códigos son equiprobables. Del mismo modo, el efecto del sesgo es mayor cuando Kappa es más pequeño. A medida que aumenta el número de códigos, los Kappa se vuelven más altos. De acuerdo con Bakeman, "ningún valor de Kappa puede considerarse universalmente aceptable". Considerando lo anterior, se han definido escalas numéricas para el Kappa, por ejemplo, Landis y Koch caracterizaron los 
Tabla 2. Total de acuerdos de los 17 observadores capacitados versus el observador experto.

\begin{tabular}{|c|c|c|c|c|c|c|c|}
\hline \multirow{2}{*}{\multicolumn{2}{|c|}{ Rango de triaje }} & \multicolumn{6}{|c|}{17 observadores capacitados } \\
\hline & & 1 & 2 & 3 & 4 & 5 & 0 \\
\hline \multirow[t]{7}{*}{ Experto } & 1 & 15 & 16 & 3 & 0 & 0 & 0 \\
\hline & 2 & 14 & 83 & 53 & 14 & 0 & 6 \\
\hline & 3 & 12 & 67 & 107 & 77 & 5 & 4 \\
\hline & 4 & 0 & 6 & 21 & 38 & 3 & 0 \\
\hline & 5 & 0 & 1 & 6 & 13 & 14 & 0 \\
\hline & 0 & 0 & 0 & 0 & 0 & 0 & 0 \\
\hline & & 41 & 173 & 190 & 142 & 22 & 0 \\
\hline \multicolumn{2}{|l|}{ Concordancias por azar } & 2,4118 & 50,882 & 89,412 & 16,706 & 1,2941 & 160,71 \\
\hline \multicolumn{2}{|l|}{ Concordancias observadas } & 15 & 83 & 107 & 38 & 14 & 257 \\
\hline \multicolumn{8}{|c|}{$\begin{array}{l}34 \text { casos por } 17 \text { evaluadores es igual a } 578 . \\
\text { Cada casillero es la suma de concordancias para cada rango evaluado (ver texto de tabla } 1 \text { ). Las concordancias esperables por azar se calcu- } \\
\text { laron de la siguiente manera: multiplicando el total de cada fila y cada columna correspondiente a cada rango evaluado: } 41 \times 34 / 578= \\
2,42 ; 173 \times 170 / 578=50,89 ; 190 \times 272 / 578=89,41 ; 142 \times 68 / 578=16,71 ; 22 \times 34 / 578=1,29 . \text { Sumatoria de concordancias por azar: } \\
160,71 \text {. Concordancias observadas totales: } 257 . \\
\text { Resultado Kappa }=0,238 . \\
\text { Para el observador experto, el coeficiente Kappa versus el sistema informático fue de } 0,69 .\end{array}$} \\
\hline
\end{tabular}

valores $<0$ como indicativos de no acuerdo y $0-0,20$ como leves, $0,21-0,40$ como regular, $0,41-0,60$ como moderado, $0,61-0,80$ como sustanciales y $0,81-1$ como casi perfecto ${ }^{(7-10)}$. Fleiss caracteriza a Kappa de acuerdo con la siguiente escala: por encima de 0,75 como excelentes; 0,40 a 0,75 como regulares a buenos, y por debajo de 0,40 como pobres. Nosotros tomamos arbitrariamente la clasificación de Fleiss.

\section{Resultados}

Se obtuvieron un total de 578 clasificaciones (17 no expertos realizaron 34 casos cada uno) cuyos resultados fueron llevados a formato de planilla electrónica tipo Excel, para su análisis ulterior. A partir de los datos generados se realizó el cálculo de concordancia a través del coeficiente Kappa entre los no expertos, el experto y el sistema informatizado (ver tabla 1, relación entre índice Kappa y fuerza de concordancia). En la comparación de concordancias entre cada observador respecto al sistema de triaje informatizado, se encontró que el observador no experto con más resultados iguales obtuvo $53 \%$ de acuerdo con el sistema informatizado de triaje (18 concordantes de 34); entre los no expertos, el que obtuvo menos resultados iguales tuvo $32 \%$ de similitud (11 concordantes de 34). En el grupo de no expertos el promedio global de concordancia fue de $41 \%$. Por otro lado, al comparar las respuestas del observador experto con el sistema se observó $79 \%$ (27/34) de resultados iguales respecto al sistema informatizado de triaje. En la tabla 1 se muestran los resultados de concordancia para cada observador no entrenado en cada categoría de triaje versus el sistema informático, la concordancia evaluada con Kappa fue de 0,19. En la tabla 2 se muestran los resultados de acuerdo en cada categoría con el observador experto, la concordancia evaluada con Kappa fue de 0,23. El observador experto versus el sistema informatizado tuvo un índice Kappa de 0,695; mientras que los observadores capacitados, comparados con el sistema informatizado y el observador experto, obtuvieron un índice Kappa de 0,19 y de 0,238 , respectivamente.

\section{Discusión}

Nuestro trabajo muestra un buen nivel de concordancia si comparamos un clasificador experto versus el sistema informatizado de triaje del Hospital de Clínicas. En cambio, no se pudo comprobar la misma concordancia cuando la comparación se realizó con observadores capacitados con cuatro horas de capacitación; la concordancia fue débil entre este grupo capacitado versus un observador experto o el propio sistema informático. El observador experto tiene un nivel de concordancia su- 
perior en relación con los observadores capacitados cuando se compara con el sistema informatizado automatizado. En cambio, no se pudo comprobar la misma concordancia cuando la comparación se realizó con observadores con cuatro horas de capacitación; la concordancia fue débil entre este grupo capacitado versus un observador experto o el propio sistema informático. Se plantea que una sesión breve de capacitación no logra una concordancia adecuada con el observador experto y con el sistema informático.

En definitiva, es la utilización de la informática automatizada en el triaje como medio de estandarización y como defensa frente a la dispersión por la aleatoriedad vinculada al factor individual y administrativo. Sin embargo, aún faltan trabajos nacionales de validación para nuestro sistema, lo cual representa un factor de dispersión.

Nuestro estudio tiene varias debilidades. En primer lugar, existe un claro sesgo de selección de observadores, lo que no permite extender este trabajo a conclusiones ulteriores. Es un estudio piloto que no permite elaborar conclusiones definitivas. En segundo lugar, se incluyen observadores con diferente entrenamiento y formación en la muestra, ya que en el grupo de 17 observadores existen médicos y enfermeros. Por otro lado, no realizamos comparaciones de los niveles de concordancia con otro estándar porque no fue el objetivo. El error aleatorio es elevado dado que el tamaño de la muestra es pequeño y no hay controles de variabilidad, por ejemplo estratificar por entrenamiento o profesión de los 17 participantes. El programa de entrenamiento que se usó para capacitar a los 17 participantes no fue evaluado previamente para determinar si era necesario un mayor período de tiempo de entrenamiento. No hay trabajos que determinen cuál es el tiempo de capacitación adecuada para considerar que el personal se encuentra entrenado.

La selección del personal no fue basada exclusivamente en quienes debieran realizar el triaje, según las recomendaciones internacionales, sino en quienes realmente lo efectivizan en dichos centros, por lo que la capacidad en la captación de la urgencia en la atención, y por lo tanto en la asignación de un nivel de prioridad, puede ser variable entre los distintos participantes.

El curso de entrenamiento que se usó para capacitar a un personal no entrenado se realizó dirigido al personal de salud en forma amplia, incluyendo enfermería ${ }^{(2,11,12)}$.

Hasta donde tenemos conocimiento, a pesar de las limitaciones de este trabajo piloto, esta sería la primera publicación realizada en Uruguay sobre triaje en una puerta de emergencia utilizando un sistema nacional informatizado automatizado. Es seguro que se requiere continuar trabajando en ello, existiendo nuevas líneas de investigación en este sentido. Como parte de la tarea de extensión universitaria, la Cátedra y el Departamento de Emergencia del Hospital de Clínicas vienen realizando jornadas de difusión, capacitación y entrenamiento en el proceso de triaje en los centros de salud que así lo soliciten, tanto a nivel público como privado.

\section{Conclusiones}

El nivel de concordancia del triaje realizado por un programa informatizado es bueno cuando se lo compara con personal capacitado como experto y entrenado en su realización; sin embargo, la concordancia con el sistema informatizado es menor cuando el personal recibe un entrenamiento de breve duración. Son necesarios estudios de mayor tamaño para confirmar definitivamente estos hallazgos.

\section{Summary}

"Triage" -the process of quickly examining patients according to their priority of treatment - is a tool that has been recognized for institutional and administrative management in the Emergency Departments ${ }^{(1)}$. Eight years after its introduction, the Clinicas Hospital has an automatized and normalized process which has become the organizational bases to address consultations in a qualified manner.

The study aimed to compare triage done by health professionals who had been trained and the one done by health professionals with no prior training of IT support, to the computerized system, comparing it with results in real time.

A higher level of agreement between trained health professionals with the results in the computerized system, when compared to professionals who lacked training was observed.

The trained observer with the most matching results achieved $55.9 \%$ of agreements with the computerized triage system ( 9 out of 34), and the observer with the least matching results obtained $32.4 \%$ of similarities (11 out of 34). Global agreement level was $41.5 \%$ in the group of professionals who were not experts. Experienced observers accounted for $79.4 \%$ of equal results and a $\mathrm{d}$ kappa index of 0.695 , whereas trained observers had 0.19 and 0.23 Kappa indexes when compared to the computerized system and the experiences observer, respectively. Therefore, we find that a short training in triage does not increase agreement when compared to the computerized system and it does increase when we compare it to triage by an experienced observer. These results should be validated in larger series of patients.

\section{Resumo}

A "triagem" -processo de classificação de pacientes por prioridades assistenciais- é uma ferramenta reconheci- 
da para a gestão assistencial e administrativa dos Departamentos de Emergência ${ }^{(1)}$. No Hospital de Clínicas, depois de oito anos de funcionamento, está disponível um processo de triagem automatizado e normalizado que funciona como base da organização para a abordagem qualificada das consultas.

O objetivo deste trabalho foi comparar a concordância da triagem realizada por pessoal de saúde treinado e não treinado previamente sem apoio informático, versus sistema informatizado, comparando os resultados em tempo real.

Observou-se um maior nível de concordância do pessoal treinado com os resultados do sistema informatizado, se comparamos com o pessoal não treinado.

O observador capacitado com mais resultados concordantes teve $55,9 \%$ de concordâncias com o sistema informatizado de triagem (19 concordantes de 34), e o que obteve menos resultados concordantes $32,4 \%$ de similitude (11 concordantes de 34$)$. No grupo de no expertos a média global de concordância foi $41,5 \%$. O observador experto teve $79,4 \%(27 / 34)$ de resultados iguais e um índice de kappa d respeito al sistema informatizado de triagem. O observador experto teve um índice de Kappa de 0,695, enquanto os observadores capacitados tiveram um índice kappa de 0.19 y 0.23 quando foram comparados com o sistema informático e o observador experimentado, respectivamente. Conclui-se que um período breve de treinamento em triagem não aumenta a concordância quando se compara com SI e com um observador experimentado. Estes resultados deveriam ser validados em series maiores de pacientes.

\section{Bibliografía}

1. Madera PG. Triaje accepted into the dictionary of the Royal Spanish Academy. Emergencias 2017; 29(1):65-6.
2. Soler W, Gómez Muñoz M, Bragulat E, Álvarez A. Triage: a key tool in emergency care. An Sis San Navar 2010; 33(1):55-68.

3. Gómez Jiménez J, Torres Trillo M, López Pérez J, Jiménez Murillo L. Sistema Español de Triaje (SET). Madrid: SEMES, 2004: 111-22.

4. Gómez Jiménez J, Chanovas Borràs MR, Miró Andreu O, Daza López M, Roqueta Ejea F, Echarte Pazos JL, et al. Triatge Estructurat als Serveis d'Urgències. Document de Posició de la Societat Catalana de Medicina d'Urgències i Emergències. Barcelona: SCMU, 2008. Disponible en: http://www.socmue.cat/Upload/Documents/5/59.pdf [Consulta: 20 marzo 2017].

5. Abad-Grau MM, Ierache J, Cervino C, Sebastiani P. Evolution and challenges in the design of computational systems for triage assistance. J Biomed Inform 2008; 41(3):432-41.

6. Gómez Jiménez J, Ramón-Pardo P, Ruda Moncada C. Manual para implementación de un sistema de Triaje para los cuartos de urgencia. Washington D.C.: OPS, 2011.

7. Fleiss JL, Cohen J, Everitt BS. Large sample standard errors of kappa and weighted kappa. Psychol Bull 1969; 72: 323-27.

8. Sim J, Wright CC. The kappa statistic in reliability studies: use, interpretation, and sample size requirements. Physic Ther 2005; 85:257-68.

9. Bakeman R, Quera V, McArthur D, Robinson BF. Detecting sequential patterns and determining their reliability with fallible observers. Psychol Methods 1997; 2:357-70.

10. Landis JR, Koch GG. The measurement of observer agreement for categorical data. Biometrics 1977; 33(1):159-74.

11. Schmitz G. Quantitative outcome of the implementation of the Canadian Triage and Severity Scale in the emergency department of a national hospital. Acta méd costarric 2016; 58(3):110-4.

12. Martínez-Segura E, Lleixà-Fortuño M, Salvadó-Usach T, Solà-Miravete $\mathrm{E}$, Adell-Lleixà $\mathrm{M}$, Chanovas-Borrás MR, et al. Competence of triage nurses in hospital emergency departments. Emergencias 2017; 29(3):173-7. 